\title{
Trial Type
}

National Cancer Institute

\section{Source}

National Cancer Institute. Trial Type. NCI Thesaurus. Code C49660.

The type of clinical trial performed e.g. efficacy, safety. 